\title{
Immunohistochemical detection of cancer-associated fibroblasts in gastrointestinal cancer as a potential prognostic biomarker of survival: meta-analysis
}

\author{
Junjie Gu, Chenyu Wang, Xiuxiu Xu, Lin Zhao, Jianfeng Zhou, Chunmei Bai^, Zhao Sun \\ Department of Oncology, Peking Union Medical College Hospital, Chinese Academy of Medical Sciences, Beijing, China \\ Contributions: (I) Conception and design: C Bai, Z Sun, J Gu; (II) Administrative support: J Zhou, L Zhao; (III) Provision of study materials or \\ patients: C Wang, J Gu; (IV) Collection and assembly of data: J Gu, C Wang, X Xu; (V) Data analysis and interpretation: J Gu, C Wang, Z Sun; (VI) \\ Manuscript writing: All authors; (VII) Final approval of manuscript: All authors. \\ Correspondence to: Chunmei Bai; Zhao Sun. Department of Oncology, Peking Union Medical College Hospital, No. 41 Damucanghutong, Xicheng \\ District, Beijing 100032, China. Email: baichunmei1964@163.com; jessiesz@126.com.
}

\begin{abstract}
Background: The association between cancer-associated fibroblasts (CAFs) and prognosis of gastrointestinal cancer patients remains controversial. The purpose of the present article is to assess the role of CAFs in gastrointestinal cancer patients by performing a meta-analysis of the clinical trials published in the literature.

Methods: Several studies were conducted to compare survival between high proportion of CAFs and low proportion of CAFs in gastrointestinal cancer patients. Search strategies were performed in PubMed, EMBASE, Medline and the Cochrane Library database. Methodological quality was assessed with the Newcastle-Ottawa Quality Assessment Scale (NOS).

Results: Among 3,763 patients enrolled in 18 studies, the pooled hazard ratio (HR) 1.64 (95\% CI, 1.43 to 1.87; $\mathrm{P}<0.001$ ) for overall survival (OS) indicated that a high proportion of CAFs was associated with shorter OS. Among 3,137 patients in 7 studies, the pooled HR 2.56 (95\% CI, 1.55 to 4.22, $\mathrm{P}<0.001$ ) for disease-free survival (DFS) exhibited that a high proportion of CAFs was associated with higher risk of recurrence. The subgroup analyses based on different tumor types showed consistent results. CAFs had no correlation with age, gender, tumor localization, TNM stage, tumor grade, and tumor type.

Conclusions: The meta-analysis indicates that high proportion of CAFs is a valuable predictor of the prognosis in gastrointestinal cancer patients, and it may provide new ideas for targeted therapy in gastrointestinal cancer patients.
\end{abstract}

Keywords: Meta-analysis; prognosis; cancer-associated fibroblasts (CAFs); gastrointestinal cancer

Submitted Jun 20, 2020. Accepted for publication Sep 30, 2020.

doi: $10.21037 /$ tcr-20-2365

View this article at: http://dx.doi.org/10.21037/tcr-20-2365

\section{Introduction}

Gastrointestinal cancers, such as esophageal, gastric, colorectal cancers, are highly fatal cancers and have become the leading cause of death worldwide. According to the American Cancer Society, new cases of digestive system tumors will represent $18.4 \%$ of all sites tumors (3.2 million/17.4 million), and deaths of digestive system tumors will represent $26.4 \%$ of all sites tumors ( 0.6 million/ 2.7 million) in USA, 2018 (1). Immunotherapy and immune checkpoint inhibitors have brought revolutionary changes to the field of oncology, especially malignant melanoma,

\footnotetext{
$\wedge$ ORCID: 0000-0003-1333-9145.
} 
lung cancer in recent years. However, gastrointestinal cancer patients do not respond to this innovative therapy. Siska et al. (2) suggested a metabolic-tumor-stroma score (MeTS) to determine the likelihood of a successful antitumor immune response, and suggested in MeTS3-MeTS4 tumors with increasing levels of lactate and acidification, targeting glycolysis and/or stromal cells might be essential to allow an effective immune response, which is of special importance in the context of cancer immunotherapy.

The tumor microenvironment (TME) is equivalent to "alimentary soil" for tumor cells. Cancer-associated fibroblasts (CAFs), one of the most common stromal cells in the TME, is considered to be a class of continuously activated, highly contractive fibroblasts with needle-like morphology, which mainly express $\alpha$-SMA, vimentin and fibroblast activation protein- $\alpha(\operatorname{FAP} \alpha)(3,4)$. CAFs play an important role in tumor metabolism, growth, metastasis, immune escape, chemotherapy resistance, immunotherapy resistance, etc. (5-7). Firstly, CAFs can promote tumor cells' proliferation by providing nutrients to surrounding tumor tissues. CAFs can produce a large amount of lactic acid, ketone body and amino acid through glycolysis. Tumor cells can ingest lactic acid, amino acid and ketone body, and utilize these energy-rich nutrients for oxidative metabolism, and then produce adenosine triphosphate $(8,9)$. Secondly, CAFs can directly or indirectly regulate tumor immunity by secreting cytokines. CAFs can secrete transforming growth factor- $\beta$, tumor necrosis factor, CC chemokine ligand, IL-4, IL-6, IL-10, prostaglandin E2, human leukocyte antigen, nitric oxide, et al, and can recruit a large number of inhibitory immune cells to tumor tissues (10), and form inhibitory immune microenvironment, which directly or indirectly regulates tumor immune. Thirdly, chemokines, cytokines and angiogenic factors secreted by CAFs can promote epithelial mesenchymal transformation, stem cell transformation of tumor cells, and promote angiogenesis. Chemokines can promote stem cell formation of tumor cells $(11,12)$, induce drug resistance $(13)$, and promote angiogenesis (14) by upregulating the expression of epithelial mesenchymal transformation related genes such as ZEB-1, CXCR4 and Snail, etc. (15). Fourthly, CAFs can secrete chemokines to promote tumor cells metastasis. Specially, PDGF-activated CAFs in colorectal cancer can promote distant metastases through stanniocalcin 1 secretion, etc. (16). In addition to releasing chemokines, CAFs-mediated TME remodeling can also promote tumor invasion and metastasis. Finally, CAFs can induce drug resistance of tumor cells. CAFs can inhibit tumor cells from ingesting antitumor drugs by improving the interstitial fluid pressure of tumor tissue (17), and can also interact with tumor extracellular matrix, thus giving rise to epithelial mesenchymal transformation of tumor cells, and ultimately resulting in chemotherapy resistance of tumor cells (18). CAFs can produce a large amount of lactic acid by converting to glycolysis, regulate the activity of proton pump on tumor cell membrane, increase the acidity of TME, and alkalize the cytoplasm of tumor cells, and thus lead to chemotherapy and immunotherapy resistance of tumor cells (19). Therefore, CAFs can promote tumor proliferation, epithelial mesenchymal transformation, immune-suppression, chemotherapy and immunotherapy resistance.

Is high proportion of CAFs in tumor stroma an efficient biomarker to differentiate gastrointestinal cancer patients who are at high or low risk of poor prognosis? Several literatus have investigated the prognostic significance of CAFs in gastrointestinal cancer patients. However, the prognostic significance of CAFs in gastrointestinal cancer patients remains controversial due to limited number of studies, enrolled patients and inconsistent results. Therefore, this study intends to further evaluate the prognostic significance of CAFs in gastrointestinal cancer patients, and to explore the relationship between CAFs and the clinicopathological characteristics of gastrointestinal cancer patients.

We present the following article in accordance with the PRISMA reporting checklist (available at http://dx.doi. org/10.21037/tcr-20-2365).

\section{Methods}

This meta-analysis adhered to the standard guidelines for meta-analyses and systematic reviews. This study was based on data from previously published studies, and therefore ethical approval was not necessary.

\section{Literature search}

Two researchers (Chenyu Wang and Junjie Gu) independently performed a comprehensive systematic search in PubMed, Embase, Web of Sciences, and the Cochrane Central Register of Controlled Trials (CENTRAL) databases. The search strategy was accordance to the guidance of the Cochrane handbook. The key words include "gastrointestinal cancer" OR "gastrointestinal cancers" OR "gastrointestinal carcinoma" OR "gastric cancer" OR 
"gastric cancers" OR "gastric carcinoma" OR "esophageal cancer" OR "esophageal cancers" OR "esophageal carcinoma" OR "colorectal cancer" OR "colorectal cancers" OR "colorectal carcinoma" OR "colon cancer" OR "colon cancers" OR "colon carcinoma” OR "rectal cancer" OR "rectal cancers" OR "rectal carcinoma”, "cancer-associated fibroblast" OR "carcinoma-associated fibroblast" OR "CAF" OR "peritumoral fibroblast" OR "activated fibroblast" OR "myofibroblast" OR "myofibroblasts". Recent conferences including American Society of Clinical Oncology (ASCO), European Society for Medical Oncology (ESMO) were also screened.

\section{Inclusion and exclusion criteria}

Inclusion criteria in this analysis were set out as follows: (I) all patients were histologically diagnosed as gastrointestinal cancer, including esophageal cancer, gastric cancer, and colorectal cancer; (II) the detection of CAFs was determined in human tissues using immunohistochemistry, including fibroblasts-associated protein (FAP), $\alpha$-smooth muscle actin $(\alpha-S M A)$ and direct CAFs signature or infiltration score; (III) patients were divided into high and low proportion groups; the relationship between CAFs detection and survival outcome was investigated; (IV) studies provided sufficient information to estimate hazard ratio (HR) about overall survival (OS) and their 95\% confidence intervals (CIs). Studies that failed to meet the inclusion criteria were excluded.

\section{Data extraction}

The relevant data were extracted independently by two reviewers (Junjie Gu and Chenyu Wang) and reached a consensus on all items. The following information was extracted from each enrolled trial: general information (name of the first author, year of publication, date of extraction, title of study and author's publication details), number of patients, clinicopathological parameters of patients, cutoff value, evaluation of CAF detection, OS, HRs and $95 \%$ CIs for OS. If the HRs were not directly reported, we contacted the authors of the primary studies for additional data. If authors did not respond, we extracted data from survival curves.

\section{Quality assessment}

A quality assessment was independently conducted for all of the enrolled studies by two investigators (Junjie Gu and Chenyu Wang) using the Newcastle-Ottawa Quality Assessment Scale (NOS), and any disagreement was resolved by discussion and consensus. The maximum possible score is nine points, and NOS scores greater than six are considered high-quality studies.

\section{Statistical analyses}

HRs and their associated 95\% CIs were pooled to analyze the prognostic value of CAFs detection in gastrointestinal cancer patients. If HRs and corresponding $95 \%$ CIs were not available, we calculated these data from available numerical data using the methods reported by Parmar et al. (20). Statistical heterogeneity was evaluated using the chi-squared test and $\mathrm{I}^{2}$. Statistically significant heterogeneity was defined as a $\mathrm{P}$ value of $<0.1$ or an $\mathrm{I}^{2}$ statistic $>50 \%$. If heterogeneity was observed, a random-effects model was used to reduce the impact of heterogeneity on the results. Otherwise, the fixed-effects model was chosen. Publication bias was assessed by Egger's and Begg's tests. All statistical analyses were performed using STATA version 14.0 (StataCorp LP, College Station, TX, USA). A P value $<0.05$ was considered to indicate statistical significance.

\section{Results}

\section{Study selection}

According to our search strategy, 768 studies were identified. Through reading the study titles and abstracts, 224 records were removed because of duplicate studies, and 490 records were removed because of non-relevance with the theme. After reviewing the full texts of the 54 potentially eligible records in detail, the following studies were excluded: studies belonging to reviews, letters, personal opinions or conference abstracts without fulltext and sufficient information about the relationship between CAFs and survival $(n=12)$, studies investigating the prognostic value of other biomarkers (not FAP, a-SMA or direct CAFs signature or infiltration score) $(n=14)$, studies using risk ratio (RR) to analyze the prognostic value of CAFs in gastrointestinal cancer patients $(n=4)$, studies using odds ratio (OR) to analyze the prognostic value of CAFs in gastrointestinal cancer patients $(\mathrm{n}=1)$, and studies providing insufficient information to estimate HRs about OS and their 95\% CIs (n=3). Eventually, 21 studies (21-41) were enrolled in this meta-analysis. A flowchart depicting the 

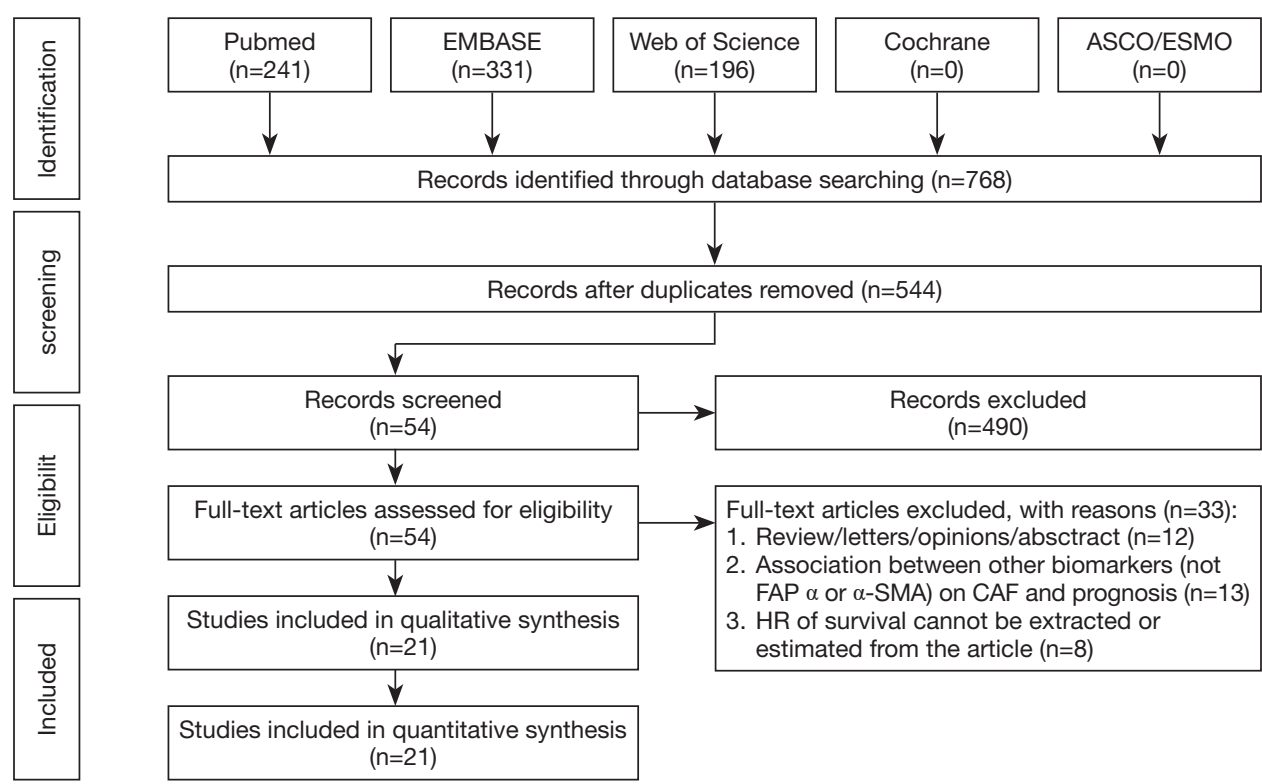

Figure 1 PRISMA flow chart of study selection.

study selection is shown in Figure 1.

\section{Study characteristics}

The characteristics of the enrolled studies are shown in Table 1 . In total, twenty studies including 6,537 patients were enrolled in the pooled analysis. The included studies were published between 2010 and 2020 in English. OS was recorded in 18 studies. Study quality, as assessed by the NOS, ranged from seven to eight. Hence, the studies were relatively high quality.

\section{Correlation between CAFs and clinicopathological characteristics}

In this present study, we investigated the association between CAFs and clinicopathological characteristics. In the enrolled 21 studies, 9 studies reported the relationship between presence of CAFs and clinicopathological features of tumors, including age, gender, tumor localization, TNM stage, tumor grade, and tumor type. Among them, 4 studies were about colorectal cancer, 3 studies about esophageal cancer, and 2 studies about gastric cancer. After analyzing the results of these 9 studies, we found it was unnecessary to perform meta-analysis to analyze the correlation between CAFs and patients' age, gender, tumor localization, TNM stage, tumor grade and tumor type, because there was no significant difference in each study (all $\mathrm{P}$ value $>0.05$ ) Therefore, we can safely draw the conclusion that there was no significant association between CAFs and gastrointestinal cancer patients' clinicopathological characteristics.

\section{CAFs as a prognostic factor for gastrointestinal cancer}

Among the 21 included articles, 18 studies involving 3,763 patients reported the relationship between CAFs and OS in gastrointestinal cancer patients. The pooled HR for OS showed that high proportion of CAFs was associated with poor OS (HR 1.64, 95\% CI, 1.43 to $1.87, \mathrm{P}<0.001$ ) in gastrointestinal cancer with a $64 \%$ increase in the risk of mortality (Figure 2). Seven articles involving 3,137 patients reported the relationship between CAFs and disease-free survival (DFS) in gastrointestinal cancer patients. The pooled HR for DFS showed that high proportion of CAFs was associated with poor DFS (HR 2.56, 95\% CI, 1.55 to $4.22, \mathrm{P}<0.001)$ in gastrointestinal cancer with a $156 \%$ increase in the risk of recurrence (Figure 3). In the subgroup analysis, the pooled HR for OS in 8 articles involving 1,846 colorectal cancer patients showed that high proportion of CAFs was associated with poor OS (HR 1.82, 95\% CI, 1.43 to $2.32, \mathrm{P}<0.001$ ) (Figure S1); 6 articles involving 1,429 gastric cancer patients showed high proportion of CAFs was associated with poor OS (HR 1.45, 95\% CI, 1.21 to 1.73 , $\mathrm{P}<0.001$ ) (Figure S2); 4 articles involving 488 esophageal 


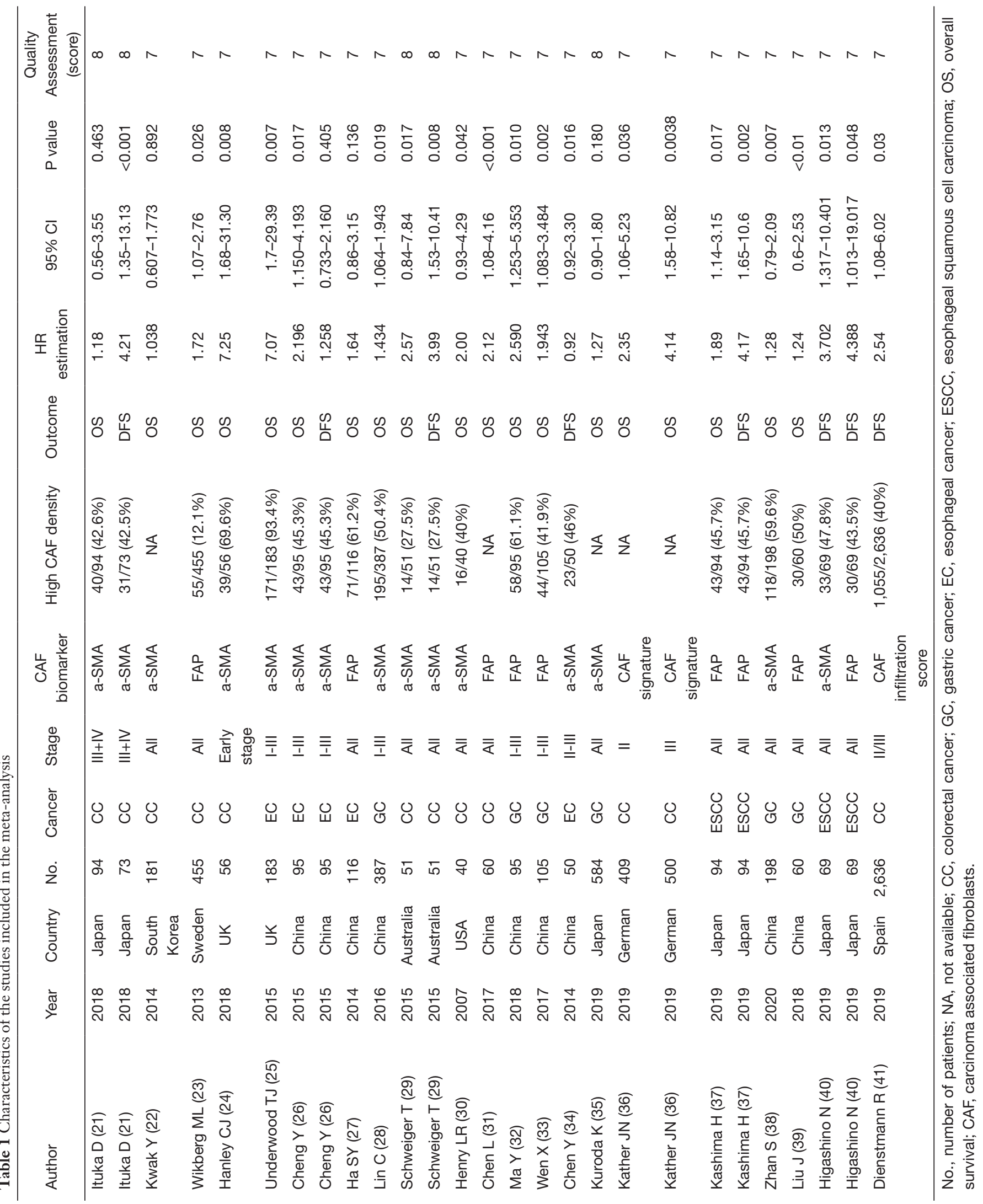




$\begin{array}{llll}\text { Study } & & & \\ \text { ID } & & & \\ \text { Wright } \\ \text { Daiji Ituka }\end{array}$

Figure 2 Forest plot describing the association between CAFs and OS in gastrointestinal cancer patients. CAFs, cancer-associated fibroblasts; OS, overall survival.

Study

ID
$\%$

HR $(95 \% \mathrm{Cl}) \quad$ Weight

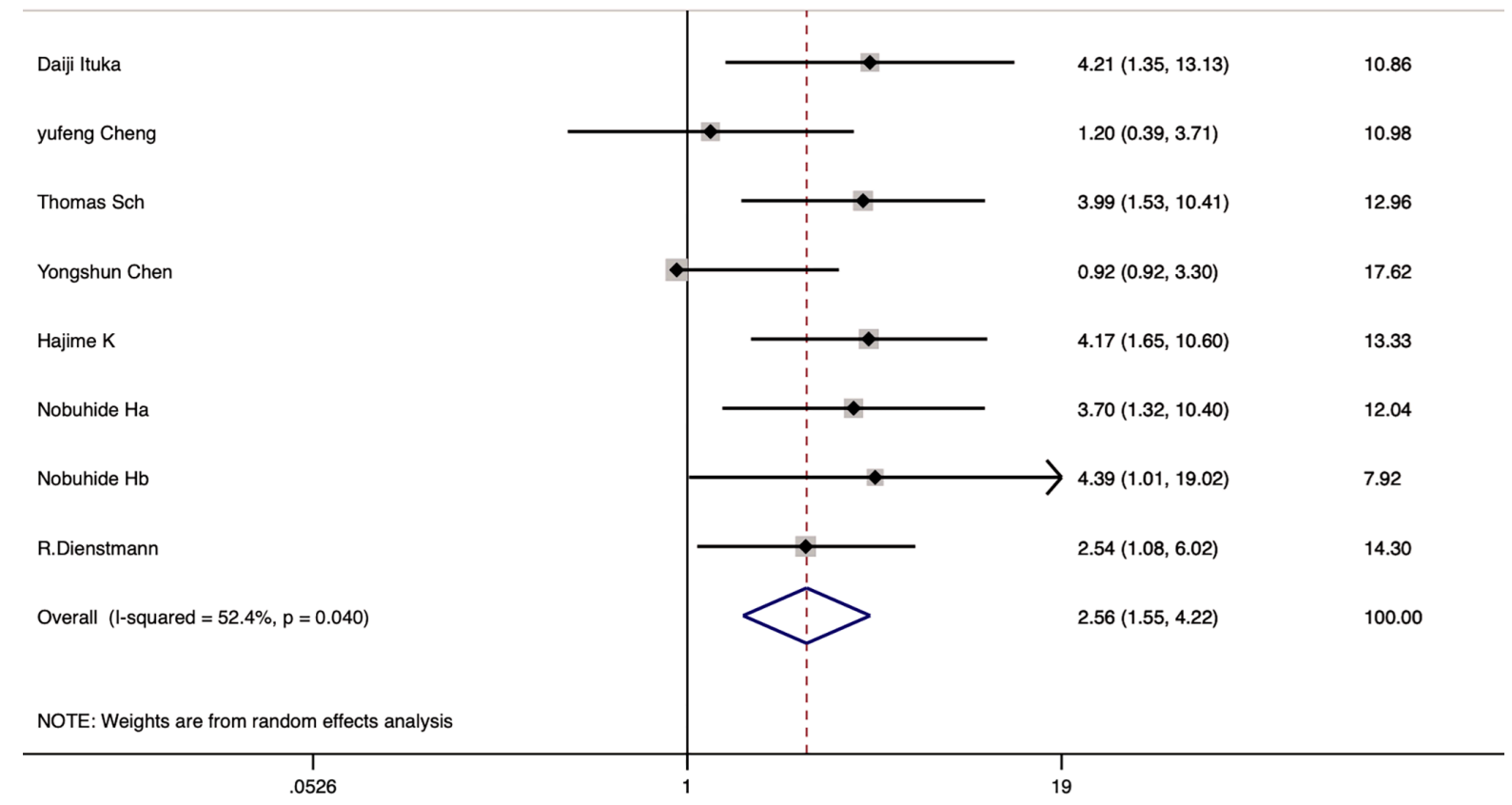

Figure 3 Forest plot describing the association between CAFs and DFS in gastrointestinal cancer patients. CAFs, cancer-associated fibroblasts; DFS, disease-free survival. 
cancer patients showed high proportion of CAFs was associated with poor OS (HR 2.03, 95\% CI, 1.46 to 2.83, $\mathrm{P}<0.001$ ) (Figure S3).

\section{Heterogeneity analysis and publication bias}

Heterogeneity observed in the analysis of correlation between CAFs and DFS $\left(\mathrm{I}^{2}=52.4 \%, \mathrm{P}=0.04\right)$. Thus, a random-effects model was employed for this analysis. The other analysis was carried out using a fixed-effects model. And both Egger's and Begg's test were applied to evaluate the publication bias in this meta-analysis. There was no evidence of publication bias shown in these studies regarding the HR and OS (Egger's test $\mathrm{P}=0.103$, Begg's test, $\mathrm{P}=0.880$ ).

\section{Discussion}

Gastrointestinal tumor is a common malignant tumor with high mortality worldwide. TNM staging (UICC/AJCC) is widely used to predict gastrointestinal cancer patients' prognosis at present. However, TNM staging cannot fully predict gastrointestinal cancer patients' prognosis due to tumor heterogeneity. Therefore, seeking new molecular predictive biomarkers is an important mission in gastrointestinal cancer field, and the relationship between TME and gastrointestinal cancer might provide new research strategies for this field.

Previous studies have demonstrated that high density of CAFs indicates poor prognosis in patients with oral squamous cell carcinoma (OSCC) (42) and breast cancer (BC) (43). This study intended to resolve the question that whether high proportion of CAFs serves as a prognostic predictor in gastrointestinal cancer patients or not. This study suggests "Yes, it does". In this study, we conducted a meta-analysis of 18 previously published high-quality studies on the correlation between CAFs and OS of gastrointestinal cancer patients, and found that high proportion of CAFs was associated with poor OS in gastrointestinal cancer patients (HR 1.64, 95\% CI, 1.43 to $1.87, \mathrm{P}<0.001$ ) with a $64 \%$ increase in the risk for mortality. A meta-analysis of 7 studies in the subgroup study showed that high proportion of CAFs was associated with poor DFS in gastrointestinal cancer patients (HR 2.56, 95\% CI, 1.55 to $4.22, \mathrm{P}<0.001$ ) with a $156 \%$ increase in the risk of recurrence. Through the study screening, quality evaluation and final meta-analysis, we strictly followed the standard guideline of Cochrane handbook, and all the data were authentic and reliable. It was thought that the heterogeneity of colorectal cancer and stomach cancer might affect the result, however, we ultimately chose gastrointestinal cancer patients as the research object in this meta-analysis due to the following reasons. They all belong to digestive tract tumors and their biology behaviors have much in common on one hand; on the other hand, the number of studies published on colorectal cancer or gastric cancer is rather limited, and the subgroup analyses based on different tumor types show consistent results. In this meta-analysis, we found no obvious heterogeneity between these 21 studies, therefore, mixed effect model was used. This was the first meta-analysis investigating the prognostic significance of CAFs in gastrointestinal cancer patients, and it clearly and objectively explicated that high proportion of CAFs was associated with poor prognosis in gastrointestinal cancer patients. However, it also had certain limitations: this study enrolled 21 studies with 6,537 gastrointestinal cancer patients, although the results were positive with statistically significant differences, the number of enrolled literatures was less still for meta-analysis.

What is the specific mechanism that high proportion of CAFs was associated with poor prognosis in gastrointestinal cancer patients? The specific mechanism could not be illuminated at present. Tumor's behavior was not entirely determined by tumor cells themselves. According to the "seed and soil" hypothesis, the tumor stroma (soil) was crucial to the development and progression of tumor cells (seeds). CAFs was an important component of TME, which were equal to activated fibroblasts in tumor stroma. As was depicted in the introduction part, many literatures had reported that CAFs could provide nutrients necessary for tumor cells' growth, directly or indirectly regulate tumor immunity, promote tumor tissue's endothelial cells' proliferation, epithelial mesenchymal transformation, and angiogenesis, promote tumor growth, invasion, chemotherapy resistance and immunotherapy resistance (4-6,12,13), and thus resulting in tumor patients' poor prognosis. Professor Song team recently reported that CD10+GPR77+ CAFs could maintain tumor cells' stemness through the continuous activation of NF- $\mathrm{KB}$ signaling pathway, thereby promoting tumorigenesis and tumor cells' chemotherapy resistance in BC and gastric cancer patients (44). Fang et al. first reported that highly metastatic hepatocellular carcinoma cells could secrete exosomes containing mir-1247-3p, leading to the continuous activation of the $\beta 1$-integrin-NF- $\mathrm{BB}$ signaling pathway in CAFs and further promoting tumor progression (45). 
However, there are few reports on the specific mechanism of CAFs regulating tumor progression in gastric cancer and colorectal cancer. Previous studies have reported that CAFs could promote the migration and invasion of gastric cancer cells by up-regulating the expression of $\beta 1$-integrin (46) or matrix metalloproteinase-2 (MMP) (47). CAFs could promote the expression of lysyl oxidase and collagen type I through the high expression of transcriptional protein 1 (Tgfb1i1) induced by the transforming factor $\beta 1$, thereby forming a microenvironment that could promote tumor growth (48). Gonçalves-Ribeiro et al. (49) found that CAFs could induce tumor cells remaining in the G0/G1 phase through the activation of PI3KCA/AKT/mTOR/Survivin signaling pathway and JAK/STAT signaling pathway, which could ensure DNA repair and accurate entrance and exit from mitosis, thus reducing the lethal effect of chemotherapy on tumor cells and leading to chemotherapy resistance. However, these studies mainly focused on a small part of the regulatory effect of CAFs, and could not yet propose a comprehensive explanation of the main regulatory mechanism that CAFs could promote poor prognosis of gastrointestinal cancer patients, nor could it provide an appropriate therapeutic target for clinical treatment, so more in-depth and complete studies are needed to fill this gap.

In conclusion, this study is the first to analyze the relationship between CAFs and the prognosis of gastrointestinal cancer patients, and found that a high proportion of CAFs was associated with poor prognosis of gastrointestinal cancer patients, which might provide new ideas for targeted therapy in gastrointestinal cancer patients. Large well-designed researches are necessary to acquire high-quality evidence.

\section{Acknowledgments}

We thank all the authors of the enrolled published papers for their valued contributions to the field.

Funding: None.

\section{Footnote}

Reporting Checklist: The authors have completed the PRISMA reporting checklist. Available at http://dx.doi. org/10.21037/tcr-20-2365

Conflicts of Interest: All authors have completed the ICMJE uniform disclosure form (available at http://dx.doi. org/10.21037/tcr-20-2365). The authors have no conflicts of interest to declare.

Ethical Statement: The authors are accountable for all aspects of the work in ensuring that questions related to the accuracy or integrity of any part of the work are appropriately investigated and resolved. This research does not involve human subjects, human materials, and therefore is not subject to approval of an institutional ethics committee.

Open Access Statement: This is an Open Access article distributed in accordance with the Creative Commons Attribution-NonCommercial-NoDerivs 4.0 International License (CC BY-NC-ND 4.0), which permits the noncommercial replication and distribution of the article with the strict proviso that no changes or edits are made and the original work is properly cited (including links to both the formal publication through the relevant DOI and the license). See: https://creativecommons.org/licenses/by-nc-nd/4.0/.

\section{References}

1. Bray F, Ferlay J, Soerjomataram I, et al. Global cancer statistics 2018: GLOBOCAN estimates of incidence and mortality worldwide for 36 cancers in 185 countries. CA Cancer J Clin 2018;68:394-424.

2. Siska PJ, Singer K, Evert K, et al. The immunological Warburg effect: Can a metabolic-tumor-stroma score (MeTS) guide cancer immunotherapy? Immunol Rev 2020;295:187-202.

3. Yang $X$, Lin $Y$, Shi $Y$, et al. FAP Promotes Immunosuppression by Cancer-Associated Fibroblasts in the Tumor Microenvironment via STAT3-CCL2 Signaling. Cancer Res 2016;76:4124-35.

4. Erez N, Truitt M, Olson P, et al. Cancer-Associated Fibroblasts Are Activated in Incipient Neoplasia to Orchestrate Tumor-Promoting Inflammation in an NFkappaB-Dependent Manner. Cancer Cell 2010;17:135-47.

5. Li X, Ma Q, Xu Q, et al. Targeting the cancer-stroma interaction: a potential approach for pancreatic cancer treatment. Curr Pharm Des 2012;18:2404-15.

6. Fang H, Declerck YA. Targeting the tumor microenvironment: from understanding pathways to effective clinical trials. Cancer Res 2013;73:4965-77.

7. El-Nikhely N, Larzabal L, Seeger W, et al. Tumor-stromal interactions in lung cancer: novel candidate targets for therapeutic intervention. Expert Opin Investig Drugs 
2012;21:1107-22.

8. Lisanti MP, Martinez-Outschoorn UE, Chiavarina B, et al. Understanding the "lethal" drivers of tumor-stroma co-evolution: emerging role(s) for hypoxia, oxidative stress and autophagy/mitophagy in the tumor microenvironment. Cancer Biol Ther 2010;10:537-42.

9. Chaudhri VK, Salzler GG, Dick SA, et al. Metabolic alterations in lung cancer-associated fibroblasts correlated with increased glycolytic metabolism of the tumor. Mol Cancer Res 2013;11:579-92.

10. Ren G, Zhao X, Wang Y, et al. CCR2-dependent recruitment of macrophages by tumor-educated mesenchymal stromal cells promotes tumor development and is mimicked by TNFalpha. Cell Stem Cell 2012;11:812-24.

11. Roccaro AM, Sacco A, Maiso P, et al. BM mesenchymal stromal cell-derived exosomes facilitate multiple myeloma progression. J Clin Invest 2013;123:1542-55.

12. McLean K, Gong Y, Choi Y, et al. Human ovarian carcinoma-associated mesenchymal stem cells regulate cancer stem cells and tumorigenesis via altered BMP production. J Clin Invest 2011;121:3206-19.

13. Liu Y, Han ZP, Zhang SS, et al. Effects of inflammatory factors on mesenchymal stem cells and their role in the promotion of tumor angiogenesis in colon cancer. J Biol Chem 2011;286:25007-15.

14. Lis R, Touboul C, Mirshahi P, et al. Tumor associated mesenchymal stem cells protects ovarian cancer cells from hyperthermia through CXCL12. Int J Cancer 2011;128:715-25.

15. Luo J, Ok Lee S, Liang L, et al. Infiltrating bone marrow mesenchymal stem cells increase prostate cancer stem cell population and metastatic ability via secreting cytokines to suppress androgen receptor signaling. Oncogene 2014;33:2768-78.

16. Peña C, Cespedes MV, Lindh MB, et al. STC1 expression by cancer-associated fibroblasts drives metastasis of colorectal cancer. Cancer Res 2013;73:1287-97.

17. Heldin CH, Rubin K, Pietras K, et al. High interstitial fluid pressure - an obstacle in cancer therapy. Nat Rev Cancer 2004:4:806-13.

18. Sui H, Zhu L, Deng W, et al. Epithelial-mesenchymal transition and drug resistance: role, molecular mechanisms, and therapeutic strategies. Oncol Res Treat 2014;37:584-9.

19. Simon S, Roy D, Schindler M. Intracellular $\mathrm{pH}$ and the control of multidrug resistance. Proc Natl Acad Sci U S A 1994;91:1128-32.

20. Parmar MK, Torri V, Stewart L. Extracting summary statistics to perform meta-analyses of the published literature for survival endpoints. Stat Med 1998;17:2815-34.

21. Ikuta D, Miyake T, Shimizu T, et al. Fibrosis in metastatic lymph nodes is clinically correlated to poor prognosis in colorectal cancer. Oncotarget 2018;9:29574-86.

22. Kwak Y, Lee HE, Kim WH, et al. The clinical implication of cancer-associated microvasculature and fibroblast in advanced colorectal cancer patients with synchronous or metachronous metastases. PLoS One 2014;9:e91811.

23. Wikberg ML, Edin S, Lundberg IV, et al. High intratumoral expression of fibroblast activation protein (FAP) in colon cancer is associated with poorer patient prognosis. Tumour Biol 2013;34:1013-20.

24. Hanley CJ, Mellone M, Ford K, et al. Targeting the Myofibroblastic Cancer-Associated Fibroblast Phenotype Through Inhibition of NOX4. J Natl Cancer Inst 2018;110:109-20.

25. Underwood TJ, Hayden AL, Derouet M, et al. Cancerassociated fibroblasts predict poor outcome and promote periostin-dependent invasion in oesophageal adenocarcinoma. J Pathol 2015;235:466-77.

26. Cheng Y, Wang K, Ma W, et al. Cancer-associated fibroblasts are associated with poor prognosis in esophageal squamous cell carcinoma after surgery. Int J Clin Exp Med 2015;8:1896-903.

27. Ha SY, Yeo SY, Xuan YH, et al. The prognostic significance of cancer-associated fibroblasts in esophageal squamous cell carcinoma. PLoS One 2014;9:e99955.

28. Lin C, Liu H, Shen Z, et al. Increased expression of aSMA is associated with poor prognosis in patients with gastric cancer after surgical resection. Int J Clin Exp Med 2016;9:11157-65.

29. Schweiger T, Nikolowsky C, Starlinger P, et al. Stromal expression of heat-shock protein 27 is associated with worse clinical outcome in patients with colorectal cancer lung metastases. PLoS One 2015;10:e0120724.

30. Henry LR, Lee HO, Lee JS, et al. Clinical implications of fibroblast activation protein in patients with colon cancer. Clin Cancer Res 2007;13:1736-41.

31. Chen L, Qiu X, Wang X, et al. FAP positive fibroblasts induce immune checkpoint blockade resistance in colorectal cancer via promoting immunosuppression. Biochem Biophys Res Commun 2017;487:8-14.

32. Ma Y, Zhu J, Chen S, et al. Activated gastric cancerassociated fibroblasts contribute to the malignant phenotype and 5-FU resistance via paracrine action in gastric cancer. Cancer Cell Int 2018;18:104. 
33. Wen X, He X, Jiao F, et al. Fibroblast Activation Proteinalpha-Positive Fibroblasts Promote Gastric Cancer Progression and Resistance to Immune Checkpoint Blockade. Oncol Res 2017;25:629-40.

34. Chen Y, Li X, Yang H, et al. Expression of basic fibroblast growth factor, CD31, and alpha-smooth muscle actin and esophageal cancer recurrence after definitive chemoradiation. Tumour Biol 2014;35:7275-82.

35. Kuroda K, Yashiro M, Sera T, et al. The clinicopathological significance of Thrombospondin-4 expression in the tumor microenvironment of gastric cancer. PLoS One 2019; 14:e0224727.

36. Kather JN, Krisam J, Charoentong P, et al. Predicting survival from colorectal cancer histology slides using deep learning: A retrospective multicenter study. PLoS Med 2019;16:e1002730.

37. Kashima H, Noma K, Ohara T, et al. Cancer-associated fibroblasts (CAFs) promote the lymph node metastasis of esophageal squamous cell carcinoma. Int J Cancer 2019;144:828-40.

38. Zhan S, Liu Z, Zhang M, et al. Overexpression of B7H3 in alpha-SMA-Positive Fibroblasts Is Associated With Cancer Progression and Survival in Gastric Adenocarcinomas. Front Oncol 2020;9:1466.

39. Liu J, Huang C, Peng C, et al. Stromal fibroblast activation protein alpha promotes gastric cancer progression via epithelial-mesenchymal transition through Wnt/ betacatenin pathway. BMC Cancer 2018;18:1099.

40. Higashino N, Koma YI, Hosono M, et al. Fibroblast activation protein-positive fibroblasts promote tumor progression through secretion of CCL2 and interleukin-6 in esophageal squamous cell carcinoma. Lab Invest 2019;99:777-92.

41. Dienstmann R, Villacampa G, Sveen A, et al. Relative contribution of clinicopathological variables, genomic

Cite this article as: $\mathrm{Gu} \mathrm{J}$, Wang $\mathrm{C}, \mathrm{Xu} \mathrm{X}$, Zhao L, Zhou J, Bai C, Sun Z. Immunohistochemical detection of cancerassociated fibroblasts in gastrointestinal cancer as a potential prognostic biomarker of survival: meta-analysis. Transl Cancer Res 2020;9(11):6629-6638. doi: 10.21037/tcr-20-2365 markers, transcriptomic subtyping and microenvironment features for outcome prediction in stage II/III colorectal cancer. Ann Oncol 2019;30:1622-9.

42. Dourado MR, Guerra ENS, Salo T, et al. Prognostic value of the immunohistochemical detection of cancer-associated fibroblasts in oral cancer: A systematic review and metaanalysis. J Oral Pathol Med 2018;47:443-53.

43. Cai D, Wu X, Hong T, et al. CD61+ and CAF+ were found to be good prognosis factors for invasive breast cancer patients. Pathol Res Pract 2017;213:1296-301.

44. Su S, Chen J, Yao H, et al. CD10(+)GPR77(+) CancerAssociated Fibroblasts Promote Cancer Formation and Chemoresistance by Sustaining Cancer Stemness. Cell 2018;172:841-56.e16.

45. Fang T, Lv H, Lv G, et al. Tumor-derived exosomal miR1247-3p induces cancer-associated fibroblast activation to foster lung metastasis of liver cancer. Nat Commun 2018;9:191.

46. He XJ, Tao HQ, Hu ZM, et al. Expression of galectin-1 in carcinoma-associated fibroblasts promotes gastric cancer cell invasion through upregulation of integrin beta1. Cancer Sci 2014;105:1402-10.

47. Yu B, Chen X, Li J, et al. Stromal fibroblasts in the microenvironment of gastric carcinomas promote tumor metastasis via upregulating TAGLN expression. BMC Cell Biol 2013;14:17.

48. Omoto T, Kim-Kaneyama JR, Lei XF, et al. The impact of stromal Hic-5 on the tumorigenesis of colorectal cancer through lysyl oxidase induction and stromal remodeling. Oncogene 2018;37:1205-19.

49. Gonçalves-Ribeiro S, Diaz-Maroto NG, Berdiel-Acer M, et al. Carcinoma-associated fibroblasts affect sensitivity to oxaliplatin and $5 \mathrm{FU}$ in colorectal cancer cells. Oncotarget 2016;7:59766-80. 